\title{
Evaluation of the quality of referral letters: experience of a Brazilian oral medicine service
}

Camila Real Delegá RODRIGUES(a) Patrícia Maria FERNANDES(a)

Alan Roger SANTOS-SILVA(a)

Pablo Agustin VARGAS(a)

Marcio Ajudarte LOPES(a)

(a) Universidade Estadual de Campinas - Unicamp, Piracicaba Dental School, Department of Oral Diagnosis, Piracicaba, SP, Brazil.
Declaration of Interests: The authors certify that they have no commercial or associative interest that represents a conflict of interest in connection with the manuscript.

Corresponding Author:

Marcio Ajudarte Lopes

E-mail:malopes@fop.unicamp.br

https://doi.org/10.1590/1807-3107bor-2021.vol35.0037

Submitted: April 15, 2020

Accepted for publication: September 9, 2020

Last revision: December 11, 2020
Abstract: Optimizing the quality of referral letters could be the first step to a correct clinical approach for patients. Referral letters should be legible and contain the necessary information to avoid delaying the patient's diagnosis and treatment. However, to date, few studies have been conducted on how referrals are performed in Brazil. Knowledge regarding the quality of referral letters can provide information to better guide health care professionals. This study was aimed at analyzing all referrals received by a single oral medicine service in Brazil. Referral letters were classified into poor, regular, good, and excellent based on key items concerning the primary morphology of the lesion, location, diagnostic hypothesis, symptomatology, time of evolution, consistency, size, and limits. A total of 500 referral letters were evaluated, including $271(54.2 \%)$ from a private service and $229(45.8 \%)$ from a public service. Dentists and physicians had written 475 (95\%) and 25 (5\%) referral letters, respectively. Among the 500 referrals analyzed in this study, the number of regular, poor, good, and excellent referrals was $236(47.2 \%)$, $203(40.6 \%), 58(11.6 \%)$, and one $(0.6 \%)$, respectively, showing a lack of important clinical information in referrals.

Keywords: Dentists; Diagnosis, Oral; Oral Medicine.

\section{Introduction}

Establishing diagnosis is an important part of the clinical process. It aims to identify the disease and allows the professional to strategize the appropriate treatment. ${ }^{1,2}$ Dentists should routinely conduct a complete examination of the patient's entire oral cavity, including all mucosal surfaces, bone, gingiva, and teeth. In the intraoral examination, a high level of knowledge-based suspicion is important to establish the early and correct diagnosis of lesions and ensure the best approach for the patient. ${ }^{3}$

For common conditions, the diagnosis is established without major difficulties. ${ }^{4,5,6}$ However, some lesions are not routinely observed in dental or medical offices and require evaluation by a professional with specific skills to reach the correct diagnosis. ${ }^{7}$ In that scenario, the patient's referral to expertise centers becomes necessary.

Professionals exchange information in different ways, but referral letters are the most common method. ${ }^{8}$ The quality of referral letters can be observed by the written information, which is the first step to an 
accurate clinical approach. Further, it should be legible and contain all the necessary information to avoid delaying the patient's diagnosis and treatment.,10

Currently, most referral letters are incomplete, not allowing the professional to receive the correct information about the patients, which may make it difficult to exactly understand the needs or concerns of those who referred them. Therefore, this study aimed to analyze all referral letters received by a single oral medicine service in Brazil.

\section{Methodology}

Referral letters were analyzed from the records of the Oral Medicine Service (Orocentro) of Piracicaba Dental School of the University of Campinas (Piracicaba, Brazil). Clinical records were reviewed from January 2016 to March 2017. A total of 500 referral letters fulfilled the inclusion criteria. All data were analyzed with Microsoft Excel (2013). The study protocol was approved by the Piracicaba Dental School Ethical Committee (protocol no. 20725719.4.0000.5418).

To evaluate the quality, referral letters were analyzed and classified as follows:

a. based on the lesion, including the primary morphology of the lesion (e.g., macule, plaque, nodule), location, size, limits, and consistency;

b. based on the history of presenting illness, including the presence/absence of symptomatology and evolution history;

c. based on the presence/absence of the diagnostic hypothesis.

A reference parameter was determined to perform classification scoring of referral letters, and each aforementioned criterion was designated one point. Thus, each referral letter received a score of points that determined the final punctuation in the score. Referral letters were classified as poor, regular, good, and excellent when scored with 0 to 1,2 to 3,4 to 6 , and 7 or 8 points, respectively (Table 1). In addition, it was verified if a dentist or physician had sent the referral and if it was from a public or private service. The inclusion criterion was the first visit for the diagnosis, and exclusion criteria were referral letters for special-needs patients, requests of conventional dental treatment for regular patients, and prior diagnosis. A total of 500 referral letters fulfilled the inclusion criteria, and data were organized according to pre-established criteria.

\section{Results}

Of all referral letters, 271 (54.2\%) came from private services and 229 (45.8\%) from public ones. Dentists had referred 475 patients (95\%), accounting for the vast majority, and physicians had referred $25(5 \%)$ patients. In terms of quality, there were 236 (47.2\%) regular, $203(40.6 \%)$ poor, $58(11.6 \%)$ good, and three $(0.6 \%)$ excellent referral letters (Table 2$)$.

When referral letters were analyzed according to the type of service (private or public) or profession (dentist or physician), they had similar classifications (Tables 3 and 4).

Considering specific items in referral letters, the location of the lesion was the most common, which was present in 433 letters (87\%). Other information more often cited included the primary morphology of the lesion $(185,37 \%)$, diagnostic hypothesis (96, $19 \%)$, symptomatology $(86,17 \%)$, time of evolution (77, $15 \%)$, consistency of the lesion $(35,7 \%)$, size $(32,6 \%)$, and limits $(28,6 \%)$. Figure and Table 5 summarize these findings.

Table 1. Classification of referral letters according to the score.

\begin{tabular}{lc}
\hline Points & Classification \\
\hline 0 or 1 & Poor \\
2 or 3 & Regular \\
4,5 , or 6 & Good \\
7 or 8 & Excellent \\
\hline
\end{tabular}

Table 2. Classification of referral letters according to the quality.

\begin{tabular}{lcc}
\hline Classification & $\mathrm{n}$ & $\%$ \\
\hline Poor & 203 & 40.6 \\
Regular & 236 & 47.2 \\
Good & 58 & 11.6 \\
Excellent & 3 & 0.6 \\
Total & 500 & 100 \\
\hline
\end{tabular}


Table 3. Classification of referral letters according to the origin .

\begin{tabular}{|c|c|c|c|c|c|}
\hline \multirow{2}{*}{ Service } & \multicolumn{4}{|c|}{ Classification } & \multirow{2}{*}{ Total } \\
\hline & Poor & Regular & Good & Excellent & \\
\hline Private & 118 & 124 & 27 & 2 & 271 \\
\hline Public & 85 & 112 & 31 & 1 & 229 \\
\hline Total & 203 & 236 & 58 & 3 & 500 \\
\hline
\end{tabular}

Table 4. Classification of referral letters according to the profession.

\begin{tabular}{|c|c|c|c|c|c|}
\hline \multirow{2}{*}{ Professional } & \multicolumn{4}{|c|}{ Classification } & \multirow{2}{*}{ Total } \\
\hline & Poor & Regular & Good & Excellent & \\
\hline Dentist & 192 & 225 & 55 & 3 & 475 \\
\hline Physician & 11 & 11 & 3 & 0 & 25 \\
\hline Total & 203 & 236 & 58 & 3 & 500 \\
\hline
\end{tabular}

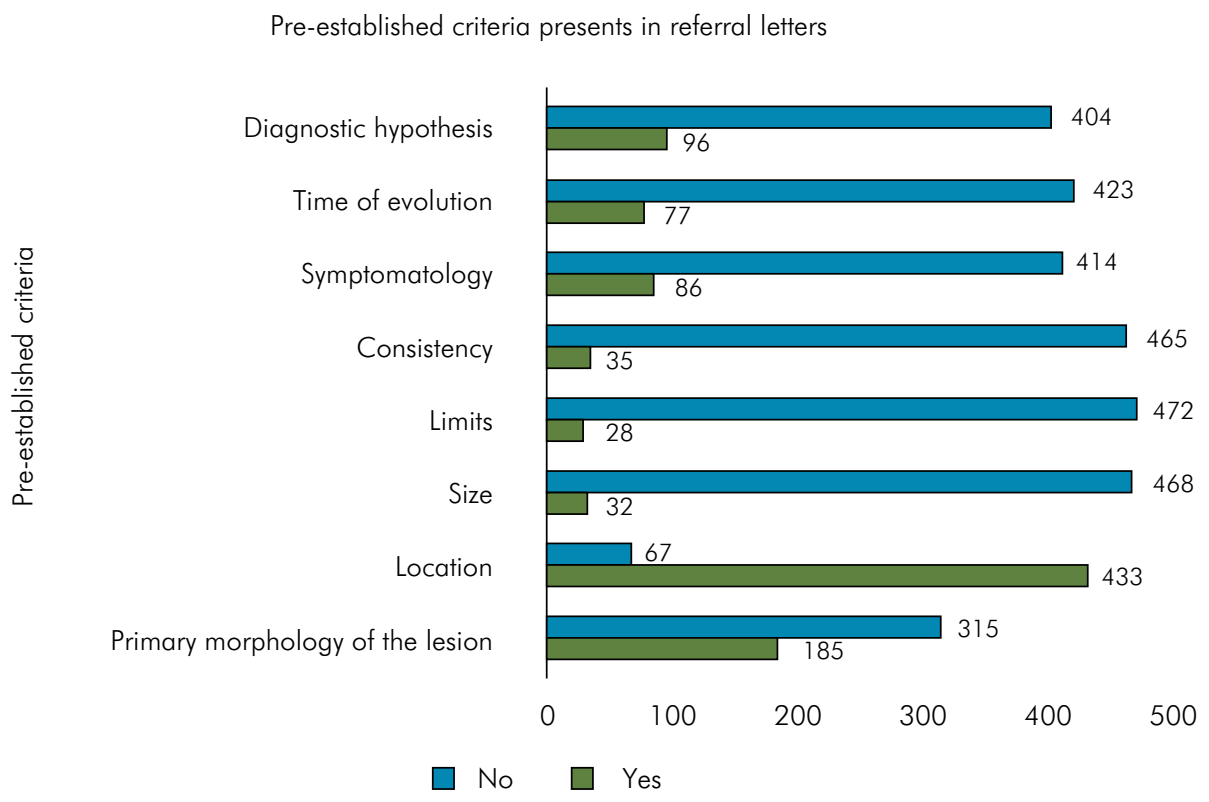

Figure. The pre-established criteria for referral letters.

Table 5. Pre-established criteria in referral letters.

\begin{tabular}{lcc}
\hline Pre-established criteria & $\mathrm{n}$ & $\%$ \\
\hline Location & 433 & $87 \%$ \\
Primary morphology of the lesion & 185 & $37 \%$ \\
Diagnostic hypothesis & 96 & $19 \%$ \\
Symptomatology & 86 & $17 \%$ \\
Time of evolution & 77 & $15 \%$ \\
Consistency & 35 & $7 \%$ \\
Size & 32 & $6 \%$ \\
Limits & 28 & $6 \%$ \\
\hline
\end{tabular}

\section{Discussion}

Referral letters are the most traditional way of communicating confidential information between two professionals. They should be of good quality and contain appropriate patient data and clinical information. When a more serious lesion is suspected, it is important to signal the need for urgency, which will help minimize the delay in diagnosis. ${ }^{11}$ However, many studies suggested this way of communication to 
often be lacking in essential information, ${ }^{12}$ contributing to miscommunication across levels of care. ${ }^{13}$ During the COVID-19 pandemic, virtual assistance to patients and health professionals, particularly dentists, could help prioritize high-risk cases. It is recommended that only patients with highly suspicious malignant lesions be referred to the oral medicine team for clinical examination and appropriate procedures, such as incisional biopsy. In that context, the delay in diagnosis and treatment is considerably longer; therefore, a well-conducted referral becomes even more important. In our study, most referral letters were classified as regular (47.2\%) or poor $(40.6 \%)$. This implies the lack of relevant and necessary information in referral letters. Previous studies have reported similar results. ${ }^{15}$

Considering the origin of referral letters (public or private service), in the current study, we observed that public and private services had similar values, although the analysis was performed in a public university. Previous studies showed that $68.6 \%$ of referral letters came from a public service, whereas only $31.4 \%$ came from a private service. ${ }^{16}$ Most professionals who referred their patients were dentists, consistent with the results of previous studies. ${ }^{16}$ This was probably because dentists more routinely see oral lesions, being their area of expertise.

The pre-established criteria chosen for this study included essential situations that provide important clinical information to the professional to assist in the diagnosis and treatment plan. Each pre-established criterion has relevance for the referral. However, the most frequent criterion included in referral letters was the location, cited in $87 \%$ (433) of referrals. Other criteria, such as symptoms, time of evolution, consistency, size, and limits of the lesion are important for the professional to formulate the diagnostic hypothesis. Therefore, the professional should be informed to include the maximum possible clinical description of lesions in the referral letters.

\section{Conclusion}

Among the 500 referral letters analyzed in this study, only 58 and 3 were classified as good and excellent, respectively. These results indicate the lack of important clinical information in referral letters. Thus, our study sheds new light on the necessity of implementing training and guidance for professionals related to the referral of patients.

\section{References}

1. Leôncio LL, Batista ÉP, Queiroz FD, Nóbrega CB, Costa LE. [ and referral of patients with oral diseases in public health services of Patos, Pernambuco, Brazil: role of the dentist in references and counter-references]. Arq Odontol. 2015;51(4):210-5. Portuguese.

2. Tommasi AF, Tommasi MH. Diagnóstico em patologia bucal. 4th ed. Rio de Janeiro: Elsevier Brasil; 2015.

3. Ali MA, Joseph BK, Sundaram DB. Dental students' ability to detect and diagnose oral mucosal lesions. J Dent Educ. 2015 Feb;79(2):140-5. https://doi.org/10.1002/j.0022-0337.2015.79.2.tb05868.x

4. Aerts D, Abegg C, Cesa K. [The role of dentists in the Unified Health System (SUS)]. Cien Saude Colet. 2004;9(1):131-8. Portuguese. https://doi.org/10.1590/S1413-81232004000100013.

5. Grimaldi N, Sarmento V, Provedel L, Almeida DD, Cunha SD. Dental care in prevention and treatment of osteoradionecrosis: literature review. Rev Bras Cancerol. 2005;51(4):319-24

6. Bertoja IC, Tomazini JG, Braosi AP, Zielak JC, Reis LF, Giovanini AF. Prevalence of oral lesions diagnosed by UnicenP Histopathology Laboratory. RSBO. 2007;4(2):41-6.

7. Vieira VG, Fernandes AM, Machado APB, Grossman SDMC, Aguiar MCF. JDevelopment alterations and lesions of the oral mucosa in patients assisted in the integrated clinics of primary attention (CIAPS) of the School of Dentistry of UFMG]. Arq Odontol. 2016;42(4). Spanish.

8. Navarro CM, Miranda IA, Onofre MA, Sposto MR. Referral letters in oral medicine: standard versus non-standard letters. Int J Oral Maxillofac Surg. 2002 Oct;31(5):537-43. https://doi.org/10.1054/ijom.2002.0277

9. Biörkeborn M, Nilsson H, Anderud J. Quality of oral surgery referrals and how to improve them. Clin Cosmet Investig Dent. 2017 Nov;9:111-6. https://doi.org/10.2147/CCIDE.S138201 
10. Zimmermann C, Meurer MI, Lacerda JT, Mello AL, Grando LJ. The use of tools to support oral lesion description in oral medicine referrals. Braz Oral Res. 2017 Nov;31(0):e93. https://doi.org/10.1590/1807-3107bor-2017.vol31.0093

11. Patel $M$, Khan $A Q$, Thiruchelvam J. Importance of quality in referral letters sent for potentially malignant oral, head and neck lesions. Dent Update. 2011 Apr;38(3):192-4. https://doi.org/10.12968/denu.2011.38.3.192

12. Akinmoladun VI, Arotiba JT, Akadiri AO. Interspecialty referrals: evaluation of quality and pattern of referral letters to an oral and maxillofacial surgery clinic. Afr J Med Med Sci. 2006 Mar;35(1):43-6.

13. Costa SM, Ferreira A, Xavier LR, Guerra PNS, Rodrigues CAQ. Referência e contrarreferência na saúde da família: percepção dos profissionais de saúde. Rev APS. 2013 Jul/Sep;16(3):287-93.

14. Lopes MA, Santos-Silva AR, Vargas PA, Kowalski LP. Virtual assistance in oral medicine for prioritizing oral cancer diagnosis during the COVID-19 pandemic. Oral Surg Oral Med Oral Pathol Oral Radiol. 2020 Jul;130(1):127-8. https://doi.org/10.1016/i.0000.2020.04.009

15. Grol R, Rooijackers-Lemmers N, Kaathoven L, Wollersheim H, Mokkink H. Communication at the interface: do better referral letters produce better consultant replies? Br J Gen Pract. 2003 Mar;53(488):217-9.

16. Navarro CM, Onofre MA, Sposto MR. Referral letters in oral medicine: an approach for the general dental practitioner. Int J Oral Maxillofac Surg. 2001 Oct;30(5):448-51. https://doi.org/10.1054/ijom.2001.0108 